\title{
Classification of Ancient Coin using Artificial Neural Network
}

\author{
Md. Iqbal \\ Quraishi \\ Dept. of Information \\ Technology \\ Kalyani Govt. \\ Engineering College \\ Kalyani, Nadia, India
}

\author{
Goutam Das \\ Dept. of Information \\ Technology \\ Kalyani Govt. \\ Engineering College \\ Kalyani, Nadia, India
}

\author{
Krishna Gopal \\ Dhal \\ Dept. of CSE \\ Kalyani Govt. \\ Engineering College \\ Kalyani, Nadia, India
}

\author{
Pratiti Das \\ Dept. of Information \\ Technology \\ Kalyani Govt. \\ Engineering College \\ Kalyani, Nadia, India
}

\begin{abstract}
Use of the coins has been started in Asia Minor during 7th century B.C. Dates back between 2500 B.C and 1700 B.C. Coins were used to trade in the Indus valley of Mohenjo-Daro and Harappa. Ancient coins are always tough to identify and recognize. Weathering and other natural causes degrades it overall structure. Classification of such ancient coins using computer vision and machine intelligence is a challenging task. Here in this paper this task has been taken to be addressed. This paper aims to develop a intelligent system which can classify and recognize ancient coins through their images only. The approach involves feature extraction classification and recognition. Standard deviation of the histogram of the image has been considered as a feature which is then classified and recognized by feed forward back propagation artificial neural network. Preprocessing of the image includes filtering of the image for better results.
\end{abstract}

\section{General Terms}

Image processing, Artificial Intelligence.

\section{Keywords}

Ancient coin, Digital image processing, Histogram, Standard deviation, feed forward back propagation artificial neural network, feature extraction, histogram analysis

\section{INTRODUCTION}

In this digital world the digital image takes an important part in many applications. [18] Digital image is a separated two dimensional matrix. Grayscale image calculate the brightness of a digital image. [10] The objects are the sets of similar pixels separated from the background through segmentation. By using segmentation, enhancement, consistency and coherent an object of the image can be identified. [5] The surface, illumination of the coin and the background of the image plays a major role to identify the coin. [9] The coin can be identifying at first by segmenting the image and then by fusing the local features and the border of the image. [19] The color, brightness, grayscale range, curves, circle of the coin can be detected by using Hough Transform and the coin can be identified. [1] The color, border, shadow of the coin to identify the coin to identify the coins by segmenting, enhancing and calculating the form factor of the binary segmentation mask. The form factor depends on the area and the perimeter of the binary mask. The image segmentation can be done by dividing the image in many regions or categories where the pixels of the same region or categories have same gray scale and neighbors from different regions have different values. [2] From gray level, color, shape, model, texture of an image, the image can be segmented regionally. [4] From the edge, the edge linking pixels the edge based segmentation is done. [6] The images of the coins are processed by an Artificial Neural Network based coin recognition system which is developed in MATLAB. The image can be trained, tested and validated by the ANN based system. [7] The gradient and the contour of the image also can be the other parameters of ancient coin segmentation. [8] By equating from coin space to Eigen face space the coin identification and classification can be done. Here Principal Component Analysis (PCA) based algorithm can be used for detection. Here we use the standard deviation of the histogram of the border image of the coins. [20] The coins have been classified by using the diameter, the shape, the gray value, the thickness, the straight lines, curves and the edge of coins as the parameters. The classification has been done by using Multilevel counter propagation neural network. [11] The clustering can be done by calculating the standard deviation of an image. Pattern recognition and classification of the objects, drawn on an image can be also done by measuring the standard deviation of any parameter of the image. Many types of projections like, vertical projection, horizontal projection are used to identify an image. [12] An image can be oriented. From projection of an image the orientation and moments also can be computed. [13] A pattern can be recognized by using the vertical and horizontal projection of an image. Here the gray value is used to calculate the values. [18] The relative frequency of each pixel which is in the gray scale image plots in the histogram. [15] Image enhancement can be done by using the histogram processing. Histogram is the graphical presentation of the gray pixel value. Image entropy of an image is the uncertainty in the image values. It can be measured by the histogram processing. Here we use artificial neural network to train the data. Artificial neural network is a processing tool which is design by using the concept of human brain and its component. [17] ANN has the intelligence to process the data and classify, recognize them. [14] An image can be classified by using ANN and detection of many features can also be done. [16] Pattern recognition technique also can be processed by ANN. There are many types of ANN. Feed forward back propagation neural network is the one of the most important type of ANN.

The proposed approach of this paper includes filtering of ancient coin images, extracting feature and classifying and recognize them using feed forward back propagation artificial neural network. Feature extraction involves histogram analysis for standard deviation value. 


\section{IMPLEMENTATION}

\subsection{Flow diagram of the method:}

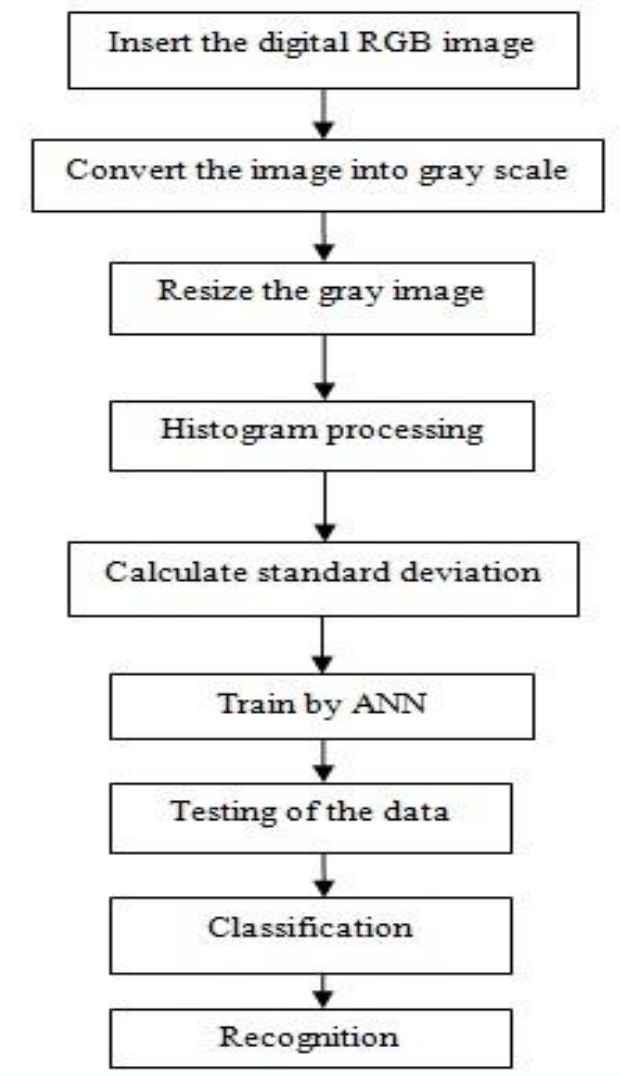

\subsection{Face Image Acquisition}

Digital RGB image is taken as the input. The test image has been shown in Fig-1

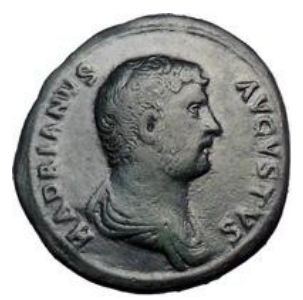

Fig-1

\subsection{Conversion to Grayscale}

The RGB image has been converted into grayscale image. The gray image is shown in Fig-2

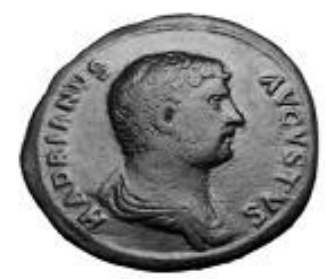

Fig.2

\subsection{Resize the gray image}

The gray image has been resized to $255 \times 255$. The resized image is shown in Fig-3.

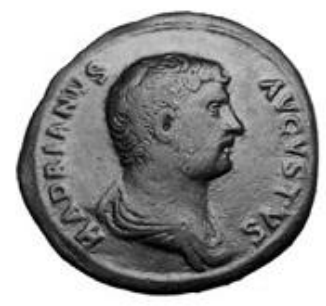

Fig-3

\subsection{Histogram processing}

Histogram process has been applied on the resized gray image. The image of the histogram of this image is shown in Fig-4.

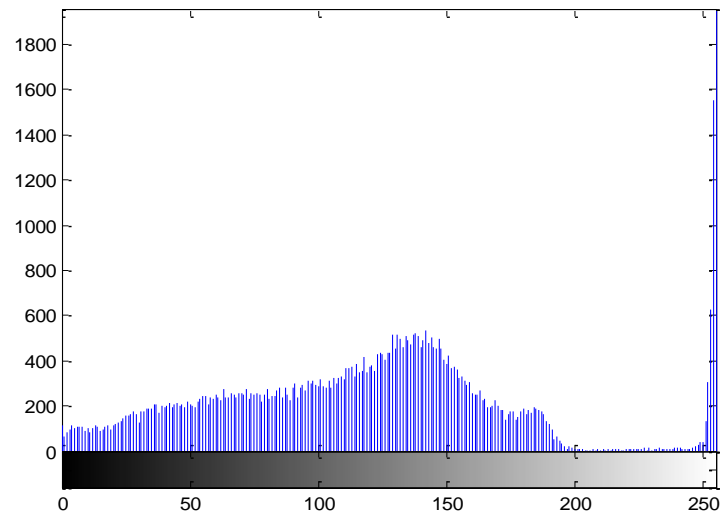

Fig.4

\subsection{Calculate standard deviation}

From the histogram the standard deviations have been measured.

\subsection{Classification using feed forward back propagation Neural Network}

An artificial neural network has $\mathrm{n}$ inputs and $\mathrm{m}$ out outputs, where $1<=\mathrm{m}<=\mathrm{n}$. The number of output nodes is equals to the number of the class of the input data. There is an intermediate layer between the input and output layers, named hidden layer. The input data is transferred from one layer to another by the neurons.

The classification system of Neural Network consisted of three stages. Training of Artificial Neural Network, Testing of Artificial Neural Network, Performance Evolution of Artificial Neural Network

\subsubsection{Training of Neural Network:}

20 images of old coins have been taken and two classes of the object drown on the images of the coins have been selected for training of the neural network. The parameters are the standard deviations of the histogram. The size of the training input matrix was $20 \times 26$.

\subsubsection{Testing of Neural Network}

After training, some coin images have been taken which has been furnished in Fig-12 for testing the Feed Forward Back 
Propagation Neural Network. The result of the testing of neural Network has been furnished in Table-1.

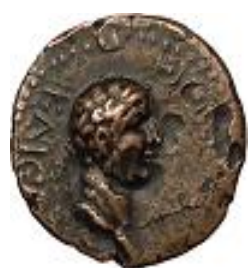

Img-1.

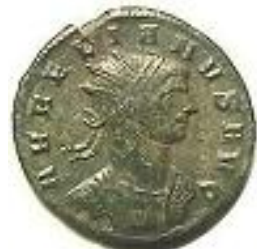

Img-4
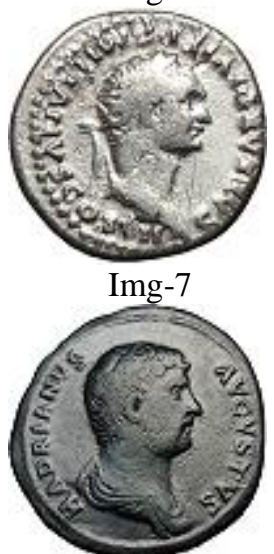

Img-10

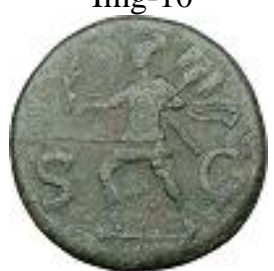

Img-13

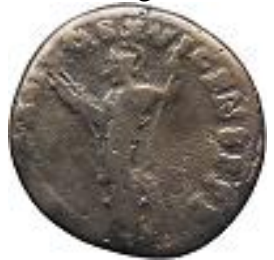

Img-16

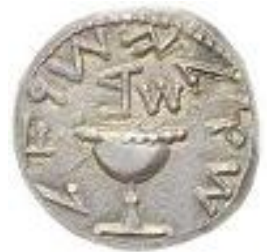

Img-19

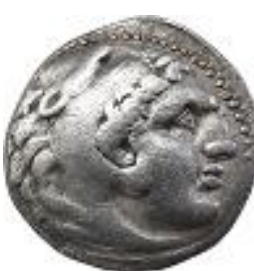

Img-2

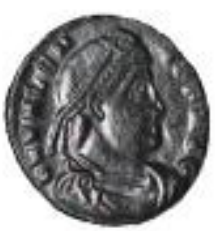

Img-5

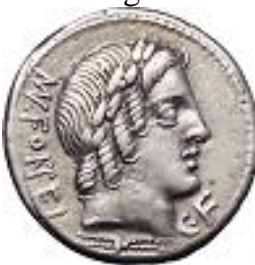

Img-8

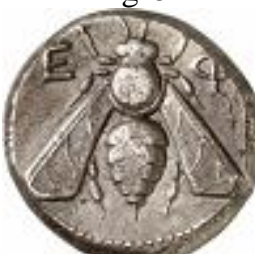

Img-11

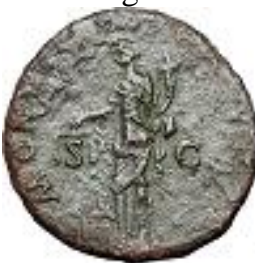

Img-14

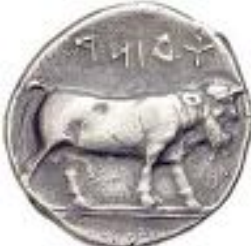

Img-17

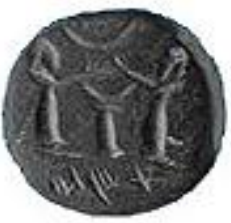

Img-20

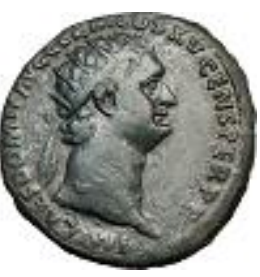

Img-3

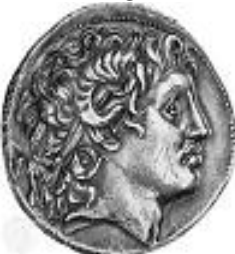

Img-6

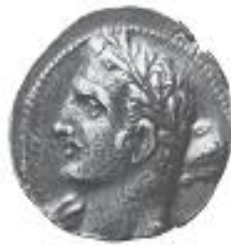

Img-9

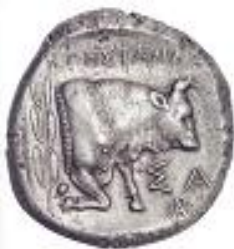

Img-12

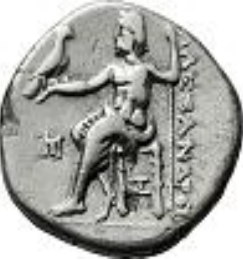

Img-15

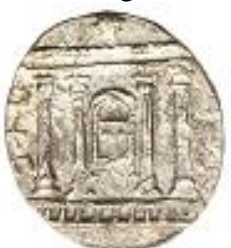

Img-18
2.7.3. Result of Testing:

Table 1

\begin{tabular}{|c|c|c|}
\hline Image & Class & Result \\
\hline 1 & 1 & Right \\
\hline 2 & 2 & Wrong \\
\hline 3 & 2 & Wrong \\
\hline 4 & 1 & Right \\
\hline 5 & 2 & Wrong \\
\hline 6 & 1 & Right \\
\hline 7 & 1 & Right \\
\hline 8 & 1 & Right \\
\hline 9 & 1 & Right \\
\hline 10 & 1 & Right \\
\hline 11 & 2 & Right \\
\hline 12 & 2 & Right \\
\hline 13 & 2 & Right \\
\hline 14 & 2 & Right \\
\hline 15 & 1 & Wrong \\
\hline 16 & 1 & Wrong \\
\hline 17 & 2 & Right \\
\hline 18 & 2 & Right \\
\hline 19 & 2 & Right \\
\hline 20 & 2 & Right \\
\hline
\end{tabular}

\subsubsection{Performance Evolution of NN:}

The performance of the neural network has been furnished in Table-2.

Table 2

\begin{tabular}{|c|c|c|c|}
\hline $\begin{array}{c}\text { Tested } \\
\text { Images }\end{array}$ & $\begin{array}{c}\text { Correct } \\
\text { recognition }\end{array}$ & $\begin{array}{c}\text { Wrong } \\
\text { recognition }\end{array}$ & $\begin{array}{c}\text { Performance } \\
(\%)\end{array}$ \\
\hline 20 & 15 & 5 & 75 \\
\hline
\end{tabular}

\section{CONCLUSION}

In this paper the study on ancient coin classification has been shown. The coins are classified into two classes according to the objects of the coins. The classification has been done by feed forward back propagation neural network using the standard deviation values from histogram approach. The testing process has been done on 20 images and the performance of the network was around $75 \%$. These results show that the classification was really effective and recognition chances are much higher. A further work can be carried out by using fuzzy logic to generate more situation specific classification and recognition in parallel or in combination with artificial neural network 


\section{REFERENCES}

[1] Sebastian Zambanini and Martin Kampel, Segmentation of Ancient Coins Based on Local Entropy and Gray Value Range",Computer Vision Winter Workshop 2008,Janez Per`s(ed.).

[2] X. Munoz, J. Freixenet, X. Cufi, J. Marti,” Strategies for image segmentation combining region and boundary information, Pattern Recognition Letters 24(2003).

[3] Fritz Albregtsen," Region \& Edge Based Segmentation of INF4300-Digital Image Analysis", page no 5-53.

[4] Sebastian Zambanini-Martin Kampel," Coin Data Acquisition for Image Rcognition".

[5] Shatrughan Modi and Dr. Seema Bawa," Automated Coin Recognition System using ANN", International Journal of Computer Applications (0975-8887) Volume 26-No.4,July 2011.

[6] Maia Zaharieva, Martin Kampel and Sebastian Zambanini." Image based recognition of coins-An overview of the COINS project.

[7] Muhammad Naveed, Rehanullah Khan, Zeeshan Khan, Syed Qasim Sattar, Yasir Ali Shah," Coins Detection Using Eigen Faces Based Upon Principal Component Analysis".

[8] Reinhold Huber-Mörk, Sebastian Zambanini, Maia Zaharieva,Martin Kampel," Identification of ancient coins based on fusion of shape and local features". Received:4August 2009/Revised:23April 2010/Accepted:20 June 2010/Published online:11July 2012.

[9] Cezar Popescu,"A Recursive Approach to Identify the Objects in a 2D Image".
[10] Bradford Bonney, Robert Ives, Delores Etter, Yingzi Du, "IRIS Pattern Extraction Using Bot Planes and Standard Deviations".

[11] Sabyasachi Dey, Bhargab B. Bhattacharya, Malay K.Kundu, Tinku Acharya, "A Simple Architecture for Computing Moments and Orientation of an Image". Fundamenta Informaticae 52 (2002) 1-11 IOS Press.

[12] Hsien-Chu Wu, Chwei-Shyong Tsai, Ching-Hao Lai, “A Licence Plate Recognition System in E-Government".

[13] C.-C. Yang, S.O. Prasher, J.-A. Landry, H.S. Ramaswamy and A. Ditommaso, "Application of artificial neural networks in image recognition and classification of crop and weeds".

[14] Komal Vij, Yaduvir Singh, "Enhancement of Images Using Histogram Processing Techniques".

[15] Amit Kumar Gupta and Yash Pal Singh, "Analysis of Back Propagation of Neural Network Method in the String Recognition".

[16] Networks for Pattern Recognition,Cambridge,TheMITPress, 1993

[17] Torsten Seemann, "Digital Image Processing Using Local Segmentation"

[18] C.M.Velu and P.Vivekanandan, "Indian Coin Recognition System of Image Segmentation by Heuristic Approach and Houch Transform (HT)"

[19] Velu C M, P.Vivekanadan, Kashwan K R, "Indian Coin Recognition and Sum Counting System of Image Data Mining Using Artificial Neural Networks". 